\title{
Metabolic Syndrome: An Interdisciplinary Approach
}

\author{
Chair: \\ Tobias Keck ${ }^{\mathrm{a}}$ \\ Participants: Marco Adamob Matthias Laudes ${ }^{c}$ Goran Marjanovic $^{d} \quad$ Beat Müller $^{\mathrm{e}}$ \\ Sebastian M. Schmidf, g Rudolf Weiner ${ }^{\text {h }}$ \\ ${ }^{a}$ Department of Surgery, University Hospital Schleswig-Holstein, Campus Lübeck, Lübeck, Germany; \\ ${ }^{\mathrm{b}}$ Bariatric Centre for Weight Management and Metabolic Surgery, University College London Hospital, London, UK; \\ ${ }^{c}$ Department of Internal Medicine, University Hospital Schleswig-Holstein, Campus Kiel, Kiel, Germany; \\ ${ }^{d}$ Center for Metabolic and Bariatric Surgery, Department of Visceral Surgery, University Hospital Freiburg, Freiburg i.Br., Germany; \\ e General, Visceral and Transplantation Surgery, Heidelberg University Hospital, Heidelberg, Germany; \\ ${ }^{f}$ Department of Internal Medicine I, University Hospital Lübeck, Lübeck, Germany; \\ ${ }^{g}$ German Center for Diabetes Research (DZD), Lübeck, Germany; \\ h Department of Obesity and Metabolic Surgery, Sana Klinikum Offenbach, Offenbach, Germany
}

\begin{abstract}
Question 1: A variety of surgical methods, malabsorptive and restrictive, are currently used in bariatric surgery. From your point of view, which is the ideal algorithm regarding the choice of procedures for overweight patients (body mass index $(\mathrm{BMI})>\mathbf{4 0} \mathrm{kg} / \mathrm{m}^{2}$ ) as well as concerning risk factors (diabetes, hypertension)? Why do you prefer the suggested algorithm?
\end{abstract}

Adamo: The ideal algorithm for choice of procedure needs to take into account:

- demographics: age, gender, BMI, patient shape, fat distribution;

- obesity-related medical conditions: type 2 diabetes mellitus (T2DM) (metabolic syndrome), obstructive sleep apnea (OSA), hypertension, polycystic ovary syndrome (PCOS), infertility, gastroesophageal reflux disease (GERD), idiopathic intracranial hypertension (IIH), etc.;

- other medical conditions: inflammatory bowel disease, pernicious anemia, kidney stones, etc.;

- medical conditions requiring lifelong pharmacological treatment which can be affected by surgery: epilepsy, rheumathoid arthritis, chronic viral infections (HIV), etc.;

- previous abdominal surgery/presence of abdominal wall herniae;

- patient reliability and compliance with vitamins and nutritional supplements;

- psychological/mental health history;

- eating habits/eating disorders.
Criteria (in order of importance) for the algorithm in choosing the procedure:

(1) patient safety;

(2) technical feasibility of the laparoscopic procedure;

(3) management of chronic conditions;

(4) patient choice;

(5) outcome.

Laudes: First step: Eating behavior: Sweet eaters should preferably receive a malabsorptive or combined procedure (e.g. bypass) instead of a purely restrictive procedure (e.g. sleeve gastrectomy).

Second step: Female subjects: Young female subjects planning future pregnancies may preferably receive a purely restrictive procedure (e.g. sleeve gastrectomy).

Third step: Comorbidities and medication:

(1) Diabetics should preferably receive a malabsorptive or combined procedure (e.g. bypass) instead of a purely restrictive procedure (e.g. sleeve gastrectomy).

(2) Subjects with GERD should preferably receive a malabsorptive or combined procedure (e.g. bypass) instead of a purely restrictive procedure (e.g. sleeve gastrectomy).

(3) Patients with severe comorbidities requiring a full drug absorption (e.g. patients with chronic inflammatory or hematological diseases or those with organ transplantation) should preferably receive a purely restrictive procedure (e.g. sleeve gastrectomy).

\section{KARGER}

(c) 2016 S. Karger GmbH, Freiburg

Fax +497614520714
Univ. Prof. Dr. med. Tobias Keck, MBA 
Marjanovic: Sleeve gastrectomy and Roux-en-Y gastric bypass (RYGB) are currently the most frequently used methods as primary procedures. So actually one has to decide between these two procedures. As both lead to equal weight loss over short time, the decision is made based upon the comorbidities of the patient as there are gastroesophageal reflux or the metabolic syndrome for gastric bypass. In super-super obese patients $\left(\mathrm{BMI}>60 \mathrm{~kg} / \mathrm{m}^{2}\right)$, a two-step strategy should be used in which sleeve gastrectomy is followed by biliopancreatic diversion. In patients with a BMI between 50 and $60 \mathrm{~kg} / \mathrm{m}^{2}$, both procedures may be used but gastric bypass should be accompanied by a second restriction such as the Minimizer band (banded bypass). Eating habits also play a role in the decision making process in patients with craniopharyngioma and central loss of satiety, but are all in all subordinate.

Müller: For overweight patients, irrespective of risk factors, we use either sleeve gastrectomy or gastric bypass as a primary surgery option. One of the two is chosen based on the patient's preference or indicated by a randomized controlled study, which is called the BariSurg trial. This constitutes our preferred algorithm because I am of the opinion that we do not yet know enough at a high level of evidence about the benefits and drawbacks of the two aforementioned worldwide most frequently used methods. Malabsorptive procedures like the single anastomosis duodenoileal bypass are used by us for redo surgery in cases of weight regain and recurrent or persisting metabolic disease.

Schmid: The choice of the right procedure in very obese patients depends on multiple individual factors and comorbidities. Unfortunately, scientific validation of the standardized algorithm is lacking. For now, we should work on the validation of such an algorithm by starting at the individual treatment goals of the patient.

Weiner: Mainly restrictive procedures (gastric bypass, sleeve gastrectomy) are the current first-line operations in the treatment of obesity and obesity-related diseases.

For the treatment of metabolic disorders, such as T2DM, bypass procedures have shown a stronger and longer-lasting effect than sleeve gastrectomy. The mini-gastric bypass (MGB)/one anastomosis gastric bypass (OAGB) has stronger effects on the metabolic syndrome (T2DM, hypertriglyceridemia, and others) than the classic proximal RYGB.

\section{Question 2: Bariatric procedures are heavily increasing in Germany as well as worldwide. How is your opinion on certification in bariatric surgery? What should a good certification system incorpo- rate?}

Adamo: Certification/accreditation in bariatric surgery is essential to achieve and maintain quality outcomes. Accreditation should be for both individual surgeons and bariatric multidisciplinary units.
For surgeons, the certification should be for each type of surgery (including primary and revisional) and should be based on:

- annual caseload;

- complication rates/mortality;

- published outcomes;

- active participation in national/international conferences.

Laudes: Infrastructure: Interdisciplinary center combining internal medicine, endocrinology, nutritional medicine, psychology, and surgery.

Staff: Internist/endocrinologist experienced in clinical bariatric patient care, at least two surgeons experienced in bariatric surgery.

Numbers: At least 100 surgical procedures per year.

Marjanovic: Certification is generally a matter of discussion and will be in bariatrics, too. Certification will not upgrade the treatment of patients in the certified centers as this is primarily dependent on the surgeons as well as their surgical skills and experience and not on some environmental factors. Centers with high case load (>100 operations/year) have automatically generated structures to deal with the high patient numbers also in the outpatient clinics. From my point of view, centers should annually publish their procedural frequency as well as the complications, i.e. morbidity and mortality, of bariatric surgery. This will show the real quality of bariatric centers as it is already performed for other procedures such as laparoscopic cholecystectomy. Quality is not a matter of a certificate but of patient outcome.

Müller: Certification is the most common form of quality control and in this way highly essential for bariatric surgery! Bariatric surgery can be easily performed but it can also become very complicated and dangerous. This is true for the procedures themselves as well as for patient care after surgery. To prevent patients from insufficient bariatric program certification systems, which should define good quality standards and well-functioning treatment processes, is very helpful.

Schmid: Obesity treatment including bariatric surgery should be performed in a multidisciplinary center with a high level of expertise on all state-of-the-art surgical methods as well as medical therapy according to guidelines. Therefore, validation and certification of interdisciplinary obesity centers is necessary to improve and maintain the highest standards and quality in the treatment of obese patients.

Weiner: Certification plays an important role in the development of specialized fields in modern medicine. Surgery for obesity and metabolic disorders is a specialized discipline, comparable with organ transplantation, for high-risk patients. The morbidity and mortality in certified centers are lower than in general departments; this has been documented in several studies.

A good certification system should incorporate the quality control system (outcome documentation) and benchmarking, documentation of continuous education and skill training, 'hardware' 
conditions (intensive care unit, operating room tables and beds for obese and superobese).

There is an important factor: the certification system should be independent but not commercial. The scientific society (for obesity surgery and metabolic disorders) has to be the highest court and control system.

\section{Question 3: Metabolic surgery for patients with a $\mathrm{BMI}<35 \mathrm{~kg} / \mathrm{m}^{2}$ is controversially discussed. What is your opinion on metabolic surgery for patients (BMI < $35 \mathrm{~kg} / \mathrm{m}^{2}$ ) concerning the correction of dia- betes mellitus?}

Adamo: I strongly advocate metabolic surgery for patients with a BMI $<35 \mathrm{~kg} / \mathrm{m}^{2}$ and T2DM. For Asian patients, surgery can be considered from a BMI of $27 \mathrm{~kg} / \mathrm{m}^{2}$.

Laudes: Since modern diabetology made huge advances in drug development (e.g. GLP1 analogs, SGLT2 inhibitors) and patient care (e.g. tight disease control), subjects with a BMI below $35 \mathrm{~kg} /$ $\mathrm{m}^{2}$ should not receive bariatric surgery.

Marjanovic: Metabolic surgery is like a rising sun but you do not know how long the day will be. A lot has been published in the past decade, and we have learned more about the development of T2DM. New therapeutic approaches have been introduced through a greater understanding of the basics. Indeed, diabetes mellitus can be brought into remission surgically and with impressive results, too. A long-term effect is not really established, unfortunately, but will be in the focus of future research. As long as there is such a successful weapon against diabetes mellitus and we do not have anything else at hand, we should use it. I think that there is no need for discussion regarding the invasiveness of surgery, such as there is no discussion on the side effects of any oral medication for any kind of disease if it is the most effective treatment. So, the interdisciplinary guidelines have to be adapted as fast as possible.

Müller: It is absolutely essential to provide access to metabolic surgery for patients with T2DM irrespective of their BMI for at least as long as their BMI is higher than $25 \mathrm{~kg} / \mathrm{m}^{2}$. The scientific evidence for the superiority of metabolic surgery over conservative treatment strategies for patients with T2DM is overwhelming. This implies that it would be unethical to withhold surgical treatment from these patients. In this regard, the BMI alone is an unpractical, outdated, and arbitrarily defined selection criterion.

Schmid: Remission of diabetes after metabolic surgery strongly depends on diabetes duration before surgery, and long-term remission rates are poor for diabetes lasting longer than 4 years.

Medical treatment for diabetes is effective and safe, and there are treatment options showing not only positive weight effects but also relevant cardiovascular benefit. There is no reason for metabolic surgery as first- or second-line therapy within an interval of $<4$ years after the diagnosis of diabetes in patients with a BMI $<35 \mathrm{~kg} / \mathrm{m}^{2}$.

Weiner: The BMI should play no role in the indication of interventions anymore. New statements of the last consensus conferences (Diabetes Summit II 2016) in London summarized: the intervention by endoscopic procedures and minimally invasive surgery should be considered if central fat distribution and other factors are existent.

Indication for surgery in the treatment of obesity for patients with T2DM in low BMI classes is included in the German guidelines.

\section{Question 4: Please provide some recommendations for the general practitioner treating a patient post restrictive bariatric surgery (e.g. sleeve gastrectomy). Which clinical and laboratory examinations should be regularly performed?}

Adamo: There are published guidelines from national and international bariatric surgical societies. Any surgical units should produce their own ones based on those and provide them to the general practitioner who is referring patients. Vitamin B12, folate, iron, vitamin $\mathrm{D}$, parathyroid hormone $(\mathrm{PTH})$, zinc, and selenium should be routinely included amongst blood tests. Vitamin B1 (thiamine) should also be considered, especially in the case of any clinical suspicion of deficiency.

Laudes: Presurgical evaluation and supplementation (if necessary) of iron, vitamin B1 (thiamine), vitamin D, vitamin B12, and folic acid.

Protein and vitamin D malnutrition is very common after bariatric surgery, including secondary hyperparathyroidism. 60-100 g/day protein and 3,000 IU/day vitamin D are recommended. Albumin is not a measure for protein supply; instead, nutritional questionnaires should be used with a calculation of nutritional protein intake.

Vitamin B1 should be supplemented in the first 3 months. Iron should be supplemented if necessary, especially in the first 6 months. In some cases intravenous supplementation is necessary.

Metformin, SGLT2 inhibitors, and GLP1 analogs should be paused within the first 6 months after surgery, and metformin should be started after 6-12 months independent of HbAlc in diabetic patients (since diabetes is not cured but in remission).

In patients with sleeve gastrectomy, gastroesophageal reflux is common. In patients with gastric bypass, lactose intolerance and dumping syndrome are common.

Marjanovic: Clinical exams: If there is pain in the upper abdomen, an ultrasound should be performed to detect gall stones. Laboratory exams: Every 6-12 months creatinine and urea and vitamin B12, calcium, and vitamin D3.

Müller: The patient should be followed up at least every 3 months during the first postoperative year and then once yearly. 
Follow-up visits should include inquiries regarding nutritional behavior, appetite, chronic vomiting, weight loss, or weight regain. They should also include screening for signs of nutritional deficiencies and depression. Additionally, weight and blood pressure should be checked as well as laboratory tests conducted that include hemoglobin, creatinine, electrolytes, vitamins, and micronutrients.

Schmid: In general, follow-up of patients after bariatric surgery - at least during the first 2 years after surgery - should be performed by specialists in internal medicine/endocrinology and diabetes with expertise in obesity and should adhere to the guidelines. However, long-term follow-up might be commissioned to the general practitioner and should include internal medicine routine (blood count, kidney and liver function, glucose metabolism, calcium and bone metabolism, thyroid function) as well as vitamin/ micronutrient status depending on the surgical method used.

Weiner: In general, there is a need for more knowledge for general physicians and other medical faculties about obesity surgery and the principles of long-term support.

Supplementation is prior to laboratory controls, and clinical symptoms are more important than X-rays and biochemical parameters. Especially knowledge of the symptoms of internal hernias after gastric bypass surgery or the beriberi disease are classic examples which show that knowledge is the most important factor for immediate and correct treatment.

\section{Question 5: Given your expertise in the field, where do you see metabolic medicine and metabolic surgery in $\mathbf{1 0}$ years from now?}

Adamo: In 10 years, metabolic surgery will be the mostly performed surgery within abdominal general surgery procedures and will become the mainstay in the treatment pathway of T2DM.

Metabolic/obesity medicine will become a medical specialty of its own (such as geriatric medicine) and will provide medical optimization for bariatric surgery.

Laudes: I would suggest that the numbers of patients treated by surgical procedures will increase; however, I do not see bariatric surgery as a 'metabolic' surgery for T2DM in subjects with a BMI < $35 \mathrm{~kg} / \mathrm{m}^{2}$.

Marjanovic: Metabolic surgery is a wide field of diagnostic and therapeutic procedures which are already performed. They will be firmly implemented in all interdisciplinary guidelines, while not being under the restriction of health insurance companies. This is the best metabolic surgery could evolve into. However, there is much more metabolic surgery can assist metabolic medicine with in understanding the development of diabetes mellitus and in establishing new targets for oral or subcutaneous medications, which can be used more individually depending on what each patient needs.

Müller: Metabolic surgery will be a common part of the treatment of metabolic disease in a multidisciplinary setting such as it is currently the case with anti-reflux surgery in the treatment of GERD, another globally spreading disease.

Schmid: Surgical techniques and methods will be further improved, and there will always be patients requiring surgical help. In parallel, there is a growing body of science in terms of disentangling the mechanisms behind the effects of metabolic surgery on human energy balance. As an example, very recent data show that the combined increase in PYY and GLP1 as seen after RYGB is key for the suppression of hunger/appetite and food intake. In contrast, GLP1 excess alone improves $\beta$-cell function in patients with diabetes, while it puts non-diabetic patients at risk for postprandial hypoglycemia due to $\beta$-cell hyperreactivity after surgery. It will be exciting to combine such mechanisms and effects with the goal of individualized medicine. There might be new concepts of medical treatment that address relevant hormonal and neuronal pathways and thereby mimic changes after metabolic surgery.

Weiner: Metabolic medicine and metabolic surgery will be a mixture of pharmaceutical, endoscopic, and minimally invasive surgery in 10 years from now.

Further investigations after obesity surgery will lead to new pharmaceutical developments and endoscopic procedures in the future. The medications and the endoscopic procedures will never be able to establish permanent (lifelong) effects. 


\section{Participants}

Prof. Marco Adamo

Bariatric Centre for Weight Management and Metabolic Surgery

University College London Hospital

Ground Floor West Wing

250 Euston Road, London NW1 2PG, United Kingdom

marcadam@doctors.net.uk

Prof. Dr. Matthias Laudes

Klinik für Innere Medizin I

Ernährungs- und Stoffwechselmedizin

Universitätsklinikum Schleswig-Holstein

Arnold-Heller-Straße 3, 24105 Kiel, Germany

Matthias.Laudes@uksh.de

Prof. Dr. Goran Marjanovic

Zentrum für Adipositas und Metabolische Chirurgie

Klinik für Allgemein- und Viszeralchirurgie

Universitätsklinikum Freiburg

Hugstetter Straße 55, 79106 Freiburg, Germany

goran.marjanovic@uniklinik-freiburg.de
Prof. Dr. med. Beat Müller

Minimal Invasive und Adipositaschirurgie

Klinik für Allgemein-, Viszeral- und Transplantationschirurgie

Universitätsklinikum

Im Neuenheimer Feld 110, 69120 Heidelberg, Germany

BeatPeter.Mueller@med.uni-heidelberg.de

Prof. Dr. med. Sebastian M. Schmid

Medizinische Klinik 1

Universitätsklinikum Schleswig-Holstein, Campus Lübeck

Ratzeburger Allee 160, 23538 Lübeck, Germany

Sebastian.Schmid@uksh.de

Prof. Dr. med. Rudolf Weiner

Klinik für Adipositaschirurgie und Metabolische Chirurgie

Sana Klinikum Offenbach

Starkenburgring 66, 63069 Offenbach, Germany

Prof.Dr.med.Rudolf.Weiner@Sana.de 